\title{
Gestão educativa: análise de uma experiência ${ }^{4}$
}

\author{
Marina Graziela Feldmann* \\ Maria Luiza Andreozzi**
}

\section{Resumo}

0 estudo refere-se à implantação do modelo estratégico-educativo de gestão, proposto e desenvolvido pela Pró- Reitoria de Graduação da PUC-SP, de 2008 a 2012. Discute conceitos que compreendem os referentes administrativos indissociáveis da participação acadêmica e da função social da PUC-SP. Interpreta a gestão como um conjunto de processos capaz de interpretar o dinamismo e a complexidade da PUC-SP, definindo e executando ações que incorporam, em si, ao mesmo tempo, movimentos de avaliação diagnóstica, de gerência de informações e de difusão do conhecimento com vistas à formação dos envolvidos. Os resultados sugerem uma relação positiva entre o modelo estratégico-educativo e 0 alcance das metas propostas no PDI - Plano de Desenvolvimento Institucional.

Palavras-chave: Gestão/PUC-SP. Modelo Estratégico-Educativo. Formação.

\section{Educational management: analysis of an experiment Abstract}

The study refers to the deployment of strategic model management-education proposed and developed by the Dean of graduate studies at PUC-SP, de 2008 a 2012. Discusses concepts that comprise those for academic and administrative inseparable from participation of the social function of PUC-SP. Interprets as a set of management processes capable of interpreting the dynamism and complexity of PUC-SP setting and performing actions that incorporate itself at the same time, diagnostic evaluation, movements of information management and dissemination of knowledge with a view to the training of those involved. The results suggest a positive relationship between the strategic model and educational-goals proposed in the PDI.

Keywords: Management/PUCSP. Education-Strategic Model. Training.

* Doutora em Educação pelo Programa de Estudos Pós-Graduados em Educação: Currículo da Pontifícia Universidade Católica de São Paulo - Professora Titular da PUCSP. E-mail: mgf@pucsp.br.

** Doutora em Educação pelo Programa de Estudos Pós-Graduados em Educação: História, Política e Sociedade da Pontifícia Universidade Católica de São Paulo - Professora Titular da PUCSP. E-mail: mlac@pucsp.br.

4 A equipe de realização deste projeto contou com a colaboração de Sandra Lucia Ferreira Acosta Soares e Ione de Carvalho. 


\section{Gestión educativa: análisis de un experimento Resumen}

El estudio se refiere a la implantación del modelo estratégico-educativo de la gestión propuesto y desarrollado por la Prorrectoría de estudios de graduación de la PUC-SP (Universidad Católica de San Pablo-Brasil), de 2008 a 2012. Discute conceptos que abarcan los referentes administrativos inseparables de la participación académica y de la función social de la PUC-SP. Interpreta la gestión como un conjunto de procesos capaz de interpretar el dinamismo y la complejidad de la PUC-SP definiendo y ejecutando acciones que incorporan, en sí, al mismo tiempo, movimientos con miras a la formación de los implicados. Los resultados sugieren una relación positiva entre el modelo estratégico-educativo y el alcance de las metas propuestas en el PDI-Plano de Desarrollo Institucional.

Palabras clave: Administración/PUC-SP. Formación. Modelo estratégico de la educación

\section{Introdução}

A Pró-Reitoria de Graduação (PROGRAD-PUC/SP) é definida, segundo o Estatuto e Regimento da Universidade, PONTIFÍCIA UNIVERSIDADE CATÓLICA DE SÃO PAULO, (2009a)5, como espaço gestor de políticas de ensino, pesquisa e extensão, implementação e acompanhamento das políticas de ensino, pesquisa e extensão, aprovadas em colegiados superiores - Conselho de Ensino, Pesquisa e Extensão (CEPE), Conselho Universitário (CONSUN) - e cuja função implica as articulações internas e externas à Universidade. A primeira função expressa a indissociabilidade entre ensino, pesquisa e extensão, proposta e orientada pelos Projetos Pedagógicos dos Cursos de Graduação, concretizadores da missão da Universidade. A segunda função manifesta essa indissociabilidade nas articulações interinstitucionais. A dimensão externa da integração entre pesquisa, ensino e extensão também se viabiliza nas propostas apresentadas em resposta às necessidades sociais, serviços e pesquisas interdisciplinares.

O ponto de partida da PROGRAD-PUC/SP surgiu da implantação do novo Estatuto e Regimento da Universidade, PONTIFÍCIA UNIVERSIDADE CATÓLICA DE SÃO PAULO (2009a), expresso nos documentos oficiais, que indicou para a PROGRAD-PUC/SP a responsabilidade pela implantação da Câmara de Graduação definida como um colegiado de Coordenadores de Cursos assim definido:

Art. 35. As Câmaras são órgãos de caráter consultivo e natureza transversal, cuja finalidade é assessorar o CEPE nas questões de sua competência, fomentando a indissociabilidade

5 Esse Estatuto de 2009 foi concebido a partir da instalação de um grupo de trabalho que tinha como meta o redesenho organizacional da PUC-SP. 
entre ensino, pesquisa e extensão. (PONTIFÍCIA UNIVERSIDADE CATÓLICA DE SÃO PAULO, 2009b, p. 91).

Assim, a organização da gestão educativa da PROGRAD-PUC/SP - 2008-2012 - estruturou a criação e implementação desse colegiado que se compunha de professores coordenadores e de representantes discentes que deviam atuar conforme o conjunto normativo de ações do Regimento Geral da Universidade:

A Câmara de Graduação é presidida pelo Pró-Reitor de Graduação e tem a seguinte composição:

I. Coordenadores de Cursos de Graduação;

II. 01 (um) representante discente dos Cursos de Bacharelado;

III. 01 (um) representante discente dos Cursos de Licenciatura;

IV. 01 (um) representante discente dos Cursos Tecnológicos.

Tendo como base a sua finalidade e para viabilizar o seu funcionamento, a PróReitoria de Graduação dividiu a Câmara de Graduação em Subcâmaras:

Subcâmara de Avaliação;

Subcâmara de Projetos Pedagógicos de Curso;

Subcâmara de Projetos, Programas e Pesquisa;

Subcâmara de Estudos e Demandas;

Subcâmara de Planejamento e Gestão Acadêmicos.

Para a realização dessa dinâmica, foi preciso, então, definir objetivos para cada uma das cinco subcâmaras, definidas em parceria com os 54 Coordenadores dos Cursos de Graduação.

Subcâmara de Avaliação: (a) apreciar os relatórios das avaliações institucionais e de cursos; e (b) promover a autoavaliação dos Cursos de Graduação das Faculdades, encaminhando ao CEPE os relatórios;

Subcâmara de Projetos Pedagógicos de Curso: (a) dar parecer sobre Projetos Pedagógicos de Cursos e propostas de alteração a serem submetidos ao CEPE; (b) subsidiar e supervisionar o desenvolvimento dos Projetos Pedagógicos dos Cursos, zelando pela observância dos mesmos; e (c) acompanhar a implementação de novos Projetos Pedagógicos de Cursos;

Subcâmara de Projetos, Programas e Pesquisa: (a) propor ao CEPE as normas e orientações técnicas para elaboração e tramitação de programas e projetos de ensino das Faculdades; e (b) emitir pareceres de mérito sobre projetos de extensão ligados à Graduação;

Subcâmara de Estudos e Demandas: Promover estudos das demandas sócio- 
ocupacionais e das transformações na esfera do conhecimento, orientando as faculdades para a proposição de mudanças curriculares ou de novas modalidades de Graduação;

Subcâmara de Planejamento e Gestão Acadêmica: promover planejamento e organização acadêmica (calendário, matrículas, infraestrutura, logística, etc.).

Nesse sentido, os Coordenadores dos Cursos de Graduação possuem a liberdade para decidir em qual subcâmara suas contribuições poderiam ser mais significativas. Assim, cada uma das subcâmaras foi composta por um grupo de Coordenadores de Cursos, cuja função estaria relacionada à abordagem das temáticas específicas de cada Subcâmara. Os cinco grupos se organizaram com a presença de um Coordenador de Subcâmara, eleito para exercer a função de organizador do trabalho da Subcâmara, por sua vez, vinculada ao trabalho da própria Câmara de Graduação.

Dessa maneira, a PROGRAD/PUC-SP - 2008-2012 - trabalhou, e ainda trabalha, com um modelo de gestão que permite a participação de todos os sujeitos envolvidos no processo de tomadas de decisões, desenvolvendo-se como ação coletiva diante de proposições, metas e desafios.

A construção da Câmara de Graduação, dadas a sua complexidade e a crescente ampliação das tarefas educativas específicas da formação inicial dos alunos, não podia ser encerrada como responsabilidade exclusiva da gestão superior. Partimos, então, do entendimento que se faz necessário à adoção de práticas interativas, participativas e democráticas, com a marca da dialogicidade, em que todos são responsáveis pela empreitada educativa em reconstrução permanente.

Nesse cenário, a Coordenação de Cursos passou a ser valorizada, tornando-se assim o núcleo estruturante da Graduação, agora com lugar específico definido no Estatuto e Regimento da Universidade, PONTIFICIA UNIVERSIDADE CATÓLICA DE SÃO PAULO (2009a), uma vez que, embora tivesse a responsabilidade de implantar e zelar pelos projetos pedagógicos de cursos, nem sempre obtinha o reconhecimento pela instituição.

A PROGRAD-PUC/SP - 2008-2012 -, de forma simultânea ao trabalho de implantação da Câmara de Graduação, iniciou seu plano com a realização de uma avaliação diagnóstica das práticas acadêmicas e 0 acompanhamento das várias dimensões institucionais já existentes reveladas pelos documentos of iciais. Essas dimensões foram assim compreendidas: (1) a estrutura e a organização dos cursos de graduação; (2) a dinâmica dos fluxos de trabalho interno; (3) as políticas de formação docente; (4) identificação dos percursos do aluno egresso e sua inserção no mercado de trabalho.

Compreender a estrutura e a dinâmica da PROGRAD-PUC/SP, bem como distinguir as condições do trabalho acadêmico - contexto social e suas complexidades - 
foram pontos nevrálgicos para a busca de um modelo que consubstanciasse as características para o binômio estratégico-educativo de gestão.

\section{A estrutura do modelo estratégico-educativo}

0 modelo estratégico-educativo de gestão foi proposto com base no entendimento dos aspectos executivos da PROGRAD-PUC/SP e dos resultados do estudo diagnóstico. A criação do modelo estratégico-educativo teve como pano de fundo exigência de transformação da PUC-SP, em direção a um aperfeiçoamento da gestão que fosse capaz de subsidiar o planejamento e a execução de ações voltadas, principalmente, para o desenvolvimento e 0 aprimoramento dos Projetos Pedagógicos dos Cursos de Graduação (PPC) e, ao mesmo tempo, pudesse oferecer condições para a aprendizagem política dos envolvidos.

Neste sentido, o enfoque foi dado à cooperação como fonte básica para a formação dos sujeitos definidos como aqueles que:

[...] se educam com o outro, quando produzem a sua existência relacionada com a existência do outro, em um processo permanente de apropriação, mediação e transformação do conhecimento mediante um projeto existencial e coletivo de construção humana (FELDM ANN, 2009, p. 72).

A definição do modelo foi baseada também numa concepção de gestão que fosse capaz de capturar, por meio de uma visão ampliada, o dinamismo e a complexidade da PUC/SP. Nesse sentido, o modelo vem sendo implementado de modo a direcionar, de forma indissociável, duas dimensões de ações: as estratégicas e as educativas.

Assim, o modelo proposto se constituiu a partir dessas duas dimensões, e se consolidou como estratégico-educativo, assumindo, portanto, a questão da indissociabilidade como traço intrínseco desse binômio; um modelo que pode ser caracterizado como abrangente e diversificado. E isso se justifica, porque, algumas vezes, aproximou-se da proposta de regulação e, dessa forma, tomou como referência caráter mais funcional e instrumental, indicando a necessidade organizacional da gestão voltada para a coordenação e a normatização. Outras vezes, o modelo enfatizou a dimensão política que se traduziu em ações menos normativas para ampliar e dar mais consistência ao debate sobre o processo decisório no âmbito da Universidade, valorizando o papel das ideias e do conhecimento.

Apesar da aparente polarização, o conceito não demonstrava incompatibilidade, muito menos excludência entre as perspectivas. Em certa medida, elas traziam dinamismo para discussões e ações associadas às prioridades descritas no Plano de Desenvolvimento Institucional. 
De um lado, consideramos estratégico o trabalho que partiu de uma proposta contextualizada e comprometida pela gestão educativa, orientada por dois pressupostos previamente estabelecidos: (1) a manutenção da qualidade acadêmica; e (2) a ampliação tanto da participação da comunidade interna como da função social da Instituição de Ensino Superior (IES). Nesse sentido, o modelo em pauta reconhecia a gestão como atividade que articularia vários setores de ação da Universidade e, por conseguinte, vários atores com diferentes funções e, portanto, com vistas diversas em relação aos propósitos institucionais.

De outro lado, tomamos como educativo, a busca da gestão da PROGRAD-PUC/ SP - 2008-2012 - orientada pela articulação dos atores em torno de uma proposta comum de trabalho, pressupondo que todos devessem se envolver enquanto sujeitos na elaboração e na execução de um projeto amplo. Um projeto que devia ser capaz de estimular o protagonismo dos sujeitos envolvidos e de lhes oferecer reconhecimento pelos resultados obtidos.

Desse modo, a concepção de envolvimento dos sujeitos devia estar articulada à compreensão de que os processos de formação caminham junto à produção de uma IES. Isso ocorrendo por meio de ações coletivas, desde a gestão, as práticas curriculares e as condições concretas de trabalho vivenciadas, que são revistas em função da dinâmica universitária. Para tanto foi necessário compreender a PUC-SP como uma comunidade educativa, composta de:

[...] vários actores e agentes locais [...], ligados entre si por relações de parceria, de programas conjuntos, de protocolos de colaboração, contribuindo para a construção de um espaço educativo congruente que resulta da aplicação de uma determinada política educativa, [...] envolvendo grande diversidade de actores e movimentos (FERNANDES, 2005, p. 193).

Previsto para ser desenvolvido no período 2008/2012, o modelo estratégicoeducativo viabilizou-se por meio de um abrangente e diversificado conjunto de conceitos e instrumentos. De fato, os sujeitos responsáveis por essa gestão da Graduação optaram por trabalhar simultaneamente com as duas dimensões, a organizacional e a política dos cursos de Graduação ao encaminhar avaliação e normatização dos cursos por meio da ampliação da participação da comunidade universitária - gestores, professores e estudantes.

Tal opção valorizava como referência o conhecimento produzido pelos sujeitos no interior do processo, além de proporcionar condições para o seu desenvolvimento contínuo da aprendizagem política. Dessa forma, seria superada a limitadora perspectiva que dissociava e polarizava, antes, gestão e produção de conhecimento; ao contrário, queríamosa concepção educativa ea participativa de gestão transformadora do processo 
de coordenação dos Cursos de Graduação, trazendo assim oportunidades para reflexões em base coletiva, valorizando e incorporando o dinamismo da vida universitária, com vistas a responder e propor prioridades para o Plano Pedagógico Institucional (PPI) e, por consequência, para o Plano de Desenvolvimento Institucional (PDI).

0 trabalho proposto partiu de uma ideia contextualizada e comprometida de gestão, orientada por pressupostos e objetivos previamente estabelecidos, como: (1) manutenção e ampliação da qualidade acadêmica dos cursos; (2) ampliação da participação da comunidade universitária; (3) aumento da capacidade institucional para responder às demandas sociais e educacionais relacionadas à sua missão.

Assim, o modelo reconhecia a gestão da Graduação como uma instância que articulava vários setores da Universidade e, portanto, integrava os vários atores e suas diferentes funções, com vistas ao desenvolvimento de projetos comuns que pudessem responder a propósitos institucionais, bem como à criação de possibilidades de transformação, à medida que se desvelassem significativas no contexto.

Pudemos observar que todo esse movimento, de deslocamento de concepção de gestão de um modelo estático para um modelo dinâmico, participativo- democrático, em que os sujeitos seformam no processo, requeria o entendimento da instituição, como uma realidade, em si mesma, inacabada, e, portanto, em constante criação, recriação e transformação.

Em síntese, o modelo proposto compreendia o sujeito como realizador de sua própria história, reconhecendo-o na gestão universitária como ator in loco para constituição do sujeito social e da ação social. Além disso, o modelo se inseria na realidade da PUC/SP e se movimentava no sentido de ser reconhecido enquanto parte de uma estrutura universitária, ou seja, enquanto parte de uma realidade específica.

Diante desses desafios, o modelo estratégico-educativo, adotado pela gestão da Graduação, desenvolveu- se na mesma ocasião em que a universidade estava (e ainda está) implantando o Redesenho Institucional, com vistas a atender e equacionar problemas institucionais inicialmente diagnosticados, como: (1) desarticulação entre unidades responsáveis por ensino, pesquisa e extensão; (2) fragmentação, paralelismo e isolamento de setores do ponto de vista das funções e dos fluxos; (3) multiplicação de instâncias que burocratizam os processos; (4) modelo de financiamento centrado em mensalidades; (5) inadequação das estruturas acadêmico-administrativas do ponto de vista da sustentabilidade financeira; (6) incoerências e inconsistências do atual estatuto, que foram sendo contornadas por portarias, resoluções e deliberações.

Nesse contexto, o modelo proposto pautou sua dinâmica também pelas necessidades requeridas por esse Redesenho Institucional, correspondendo ao atendimento de valores de fundo para a Universidade: (1) a democracia interna; 
(2) a educação humanista e o compromisso social; (3) a indissociabilidade entre ensino, pesquisa e extensão; e (4) a dimensão plural, crítica e participativa da comunidade universitária.

\section{A dinâmica da gestão com base no modelo estratégico-educativo}

A dinâmica da gestão com base no modelo estratégico-educativo ocorreu como uma forma favorecedora para implantar a PROGRAD-PUC/SP - 2008- 2012 -, por meio do desenvolvimento de projetos/ações capazes de interagir com diferentes setores da Universidade, propiciando um espaço intersubjetivo de obtenção, criação, troca e divulgação de conhecimentos que, ao final, atendessem aos interesses dos Cursos de Graduação com vistas à produção de um trabalho mais eficiente, eficaz e com informações mais precisas. Algo que teve (e ainda tem) como desafio o investimento na formação de profissionais envolvidos, reconhecendo a capacidade e responsabilidade de todos em consolidar e avançar um projeto coletivo a partir do interior desta Universidade.

Para tanto, os diferentes projetos da PROGRAD-PUC/SP - 2008-2012 - assentam-se em três procedimentos estratégicos que foram trabalhados de forma articulada. São eles: (1) a avaliação, (2) a gerência da informação e (3) a difusão de conhecimento.

No que tange à avaliação, sabemos que se trata de uma ação fundamentada em valores humanos, exercida por meio de conhecimentos científicos, filosóficos, culturais; e que é pautada, por sua vez, pela busca do aperfeiçoamento humano. Trata-se de procedimento estratégico imprescindível à potencialização de recursos disponibilizados e bastante necessários à implementação da política de educação da Universidade e a resolução de problemas. Tem o propósito de revelar questões que, de outra forma, não poderiam ser identificados; iluminar aspectos que demandam mudanças e esclarecer os envolvidos da necessidade dessas mudanças. Possui como propósito, ainda, informações confiáveis para a tomada de decisão com vistas ao aperfeiçoamento do trabalho desenvolvido nos Cursos de Graduação.

No referente à gerência da informação, consideramos aí uma demanda da atualidade, em que o diferencial de um trabalho está na sua capacidade de sistematizar, organizar e produzir conhecimento. Dessa forma, administrá-lo (armazená- lo e compartilhá-lo) tornou-se uma tarefa fundamental. Os dados disponíveis na universidade são, sem dúvida, parte integrante do seu patrimônio, pois é considerado como um importante recurso que procura atender às necessidades de informações inerentes ao nível estratégico das diferentes demandas da comunidade universitária. Nesses termos, o trabalho da PROGRAD-PUC/SP - 2008-2012 - , em parceria com a Divisão de Tecnologia da Informação (DTI), consistiu em operar as informações disponíveis na PUC-SP, viabilizando o acesso ágil, eficiente e 
seguro. Uma vez sistematizadas e operacionalizadas as informações, constituíram importantes fontes de consulta e de conhecimento, indispensáveis às atividades de análise de resultados, monitoramento e tomadas de decisões.

Em relação à adoção da difusão de conhecimento, quisemos gerar a aproximação entre os trabalhos desenvolvidos pela administração de informações. A difusão do conhecimento requereu planejamento técnico para sua execução, que, por consequência, demandava a adoção de princípios para finalizar e iniciar, ao mesmo tempo, um processo cíclico de ação, no qual o conhecimento devia ser devolvido para a comunidade (legitimação) que, ao avaliá-lo, iniciava um novo processo avaliativo, a ser aperfeiçoado pela administração de novas informações. A difusão do conhecimento, como princípio, implicava a necessidade de criação de processos de validação, pela comunidade, que participava de forma reflexiva, reiterando os estudos realizados ou indicando a necessidade de outros estudos.

A título de exemplificação, segue abaixo a apresentação de dois projetos, desenvolvidos pela PROGRAD-PUC/SP - 2008-2012 -, concebidos e realizadosa partir da articulação dos três eixos, que incorporam fundamentalmente nossa proposição.

\section{Projetos Pedagógicos dos Cursos: acompanhamento e normatização}

0 acompanhamento dos trabalhos de conversão da carga horária de horas-aula para horas-relógio proporcionou a oportunidade para quea PROGRAD-PUC/SP pudesse desenvolver simultânea e articuladamente os processos de avaliação e normatização dos Cursos de Graduação da PUC-SP, de acordo com sua concepção de gestão educativa e participativa.

De fato, os PPCs não são apenas documentos que formalizam uma proposta de curso orientada pelas Diretrizes Curriculares e subordinada à legislação. Além disso, eles podem e devem ser a expressão da forma como os proponentes e atores institucionais concebem e desenvolvem o processo de produção e difusão do conhecimento; entendem e praticam a articulação entre teoria e prática; desenvolvem a interdisciplinaridade, a pesquisa, o ensino, a extensão; e entendem as relações entre Universidade e Sociedade.

Por outro lado, os PPCs implicam a existência de um complexo sistema institucional que deve atender tanto às necessidades específicas do desenvolvimento de cada curso, quanto do conjunto deles, no contexto da PUC-SP e de sua proposta, concentrada em: estruturas relacionadas ao registro acadêmico, à gestão acadêmica e à financeira.

Por essa razão, os PPCs são simultaneamente o registro e a expressão de um processo vivo e dinâmico, que deve ser constantemente avaliado, revisto e atualizado. Nesse sentido, 
de acordo com sua natureza, os PPCs informam todo o processo de realização dos cursos, apresentando-se como um grande desafio para a comunidade universitária, ao garantir a participação específica de todos os atores na construção do projeto de formação e integrar a dinâmica da participação ao fluxo e ao registro de informações- do vestibular à certificação.

Durante o período de 2009 a 2011, a PROGRAD- PUC/SP coordenou o trabal ho dos diferentes setores envolvidos na atualização dos PPCs dos 54 Cursos de Graduação. Além de realizar diferentes modalidades de ajustes nos projetos pedagógicos, com vistas à normatização dos cursos e suas propostas acadêmicas, o processo proporcionou a oportunidade para o diálogo entre os setores acadêmico, administrativo e financeiro da instituição. Em todos os setores foram feitos ajustes de funcionamento para garantir clareza e precisão das informações, de tal forma que os PPCs, entendidos em sua dinâmica acadêmica, contribuíram para a discussão de setores específicos, que se comprometeram a realizar ajustes com vistas a orientar processos internos - revisão do Manual do Vestibular, do Registro Acadêmico, das páginas dos sites das Faculdades, das propostas e veículos de divulgação do diferencial PUC, da certificação - e subsidiar processos externos - credenciamento, recredenciamento, avaliação e financiamento.

Ao enfrentar o desafio de rever práticas cristalizadas e atualizá-las à luz das diretrizes institucionais, a comunidade acadêmica, como um todo, dinamizou-se persistindo na tarefa de garantir a coerência entre registros (internos e externos), a prática acadêmica e educativa, a excelência na formação e os direitos dos alunos.

\section{Projeto de Autoavaliação de Cursos de Graduação}

0 Projeto de Autoavaliação de Cursos foi elaborado pela PROGRAD-PUC/SP, por meio da Subcâmara de Avaliação, para atender, assim, tanto às prioridades definidas por ela mesma, como para acatar as exigências estatutárias da PUC-SP.

O objetivo era buscar o conhecimento dos diferentes Cursos de Gradação, procurando identificá-los nas características, além de promover a correção de rumos e no investimento para melhoria dos desempenhos. Nesse sentido, como hipótese, os resultados avaliativos iriam subsidiar o trabalho desenvolvido pelos Cursos de Graduação, por meio da identificação dos desafios e no encaminhamento de soluções alternativas de problemas de diferentes naturezas.

À realização do projeto, os objetivos foram assim definidos:

subsidiar e acompanhar o trabalho avaliativo das Coordenações de Cursos nas suas diferentes etapas (elaboração, sensibilização e implementação do processo avaliativo, divulgação dos resultadose monitoramento da tomada de decisão); desencadear o processo de institucionalização da autoavaliação dos Cursos de Graduação da Universidade. 
Considerando o trabalho avaliativo como uma tarefa para a comunidade colaborativa, nos diferentes segmentos que trabalham juntos com o objetivo de melhoraria contínua na Universidade, a Subcâmara de Avaliação buscou conhecer inicialmente a experiência da Comissão Própria de Avaliação (CPA-PUC-SP) que, desde 2005, vem realizando a Autoavaliação Institucional, respondendo, inclusive junto ao INEP, sobre os resultados desse processo. Nesse trabalho de Autoavaliação Institucional, a CPA reconheceu que a

[...] avaliação é imprescindível ao desenvolvimento dos
Projetos Pedagógicos de Curso (PPC) de Graduação da
universidade, independentemente da fase em que estes
se encontram, porque ela deve ser considerada como
uma necessidade intrínseca dos projetos educacionais
(PONTIFÍCIA UNIVERSIDADE CATÓLICA DE SÃO PAULO,
2009c, p. 257).

A parceria entre a PROGRAD-PUC/SP, por meio da sua Subcâmara de Avaliação, e a CPA para o desenvolvimento de um trabalho conjunto reafirma uma preocupação prevista no Sistema de Avaliação da Educação Superior (SINAES). Propõe integrar à Autoavaliação Institucional o desenvolvimento da Avaliação de Curso, com a meta de apreender "a qualidade do curso no contexto da realidade institucional no sentido de formar cidadãos conscientes e profissionais responsáveis e capazes de realizar transformações sociais". (Instrumento de Avaliação de cursos de graduação) CONAES (2010, p. 10).

Para que a Subcâmara de Avaliação iniciasse seu trabalho, a PROGRAD solicitou que a CPA disponibilizasse um estudo longitudinal, constituído por indicadores de qualidade com informações referentes aos Cursos de Graduação da PUC-SP, geradas nos últimos dez anos. Foram encontrados: demanda, perfil docente, trajetórias dos estudantes, resultados do ENADE e custo. Afora isso, outras informações foram solicitadas para diferentes setores da universidade - Coordenadoria de Vestibulares e Concursos, pelo Departamento de Recursos Humanos, pela Secretaria de Administração Escolar (SAE) e pela Controladoria - e que serviram de base para a elaboração de diferentes relatórios institucionais. Além de proporcionar interface com 0 trabalho de outras Subcâmaras, o estudo foi considerado pela Subcâmara de Avaliação um valioso dispositivo para a construção de um diálogo inicial com os Coordenadores, imprescindível ao processo de sensibilização e de institucionalização propriamente dita da avaliação de cursos. Tal procedimento colaborativo pôde dinamizar o processo de análise e de reflexões sobre os dados e, ao mesmo tempo, orientou encaminhamentos para tomadas de decisões dos Coordenadores e equipes. Porém, evidentemente, os indicadores trabalhados nessa etapa não esgotaram o processo de avaliação de cursos. 
A Figura abaixo mostra de forma sintética a proposta descrita:

Figura 1 - Auto-avaliação dos cursos de graduação - Prograd - 2009-2010

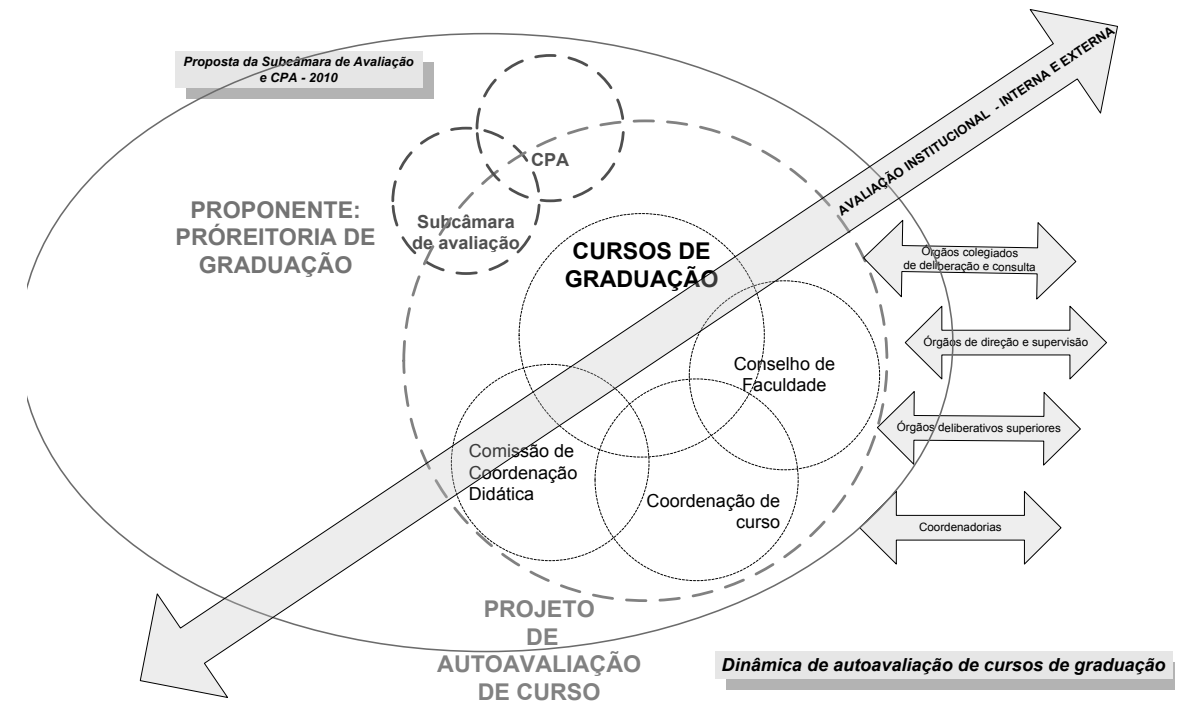

Fonte: Relatório da Comissão de Avaliação da Câmara de Graduação da Pró Reitoria de Graduação sobre a auto-avaliação dos cursos de graduação, 2010, p.8.

As estratégias para o desenvolvimento do Projeto de Autoavaliação podem ser assim vistas, ressaltando a necessidade de integrar as ações avaliativas no sistema organizacional da Universidade, de modo a gerar um processo que articule atividades analíticas às de gestão, permitindo que a avaliação subsidie, cada vez mais, decisões participativas.

Além dos resultados subjetivos evidenciados como maior motivação dos coordenadores e alunos, reforçando assim a ideia de pertencimento no processo de avaliação, vimos igualmente uma melhoria significativa não apenas em relação às mudanças efetivas em todos os cursos (vide site PUC-SP / Graduação), como na resposta formalizada por parte do INEP/MEC, quando da avaliação in loco dos referidos cursos, apresentando 80\% no patamar entre "muito bom" e "excelente".

\section{Considerações finais}

Ao longo desse período de 2008 a 2012, as condições político-acadêmicas da Universidade vêm sofrendo alterações de relevância. Algumas apresentadas nesse texto potencializaram a realização do modelo estratégico-educativo de gestão, concebido e empreendido pela PROGRAD-PUC/SP. 
O desafio hoje para a PROGRAD-PUC/SP é continuar reestruturando toda uma base produtiva constituída a partir da implantação do modelo em tela, seguindo os três grandes objetivos propostos no seu Plano de Desenvolvimento Institucional: (1) ampliar o nível de excelência acadêmica da Universidade; (2) fortalecer ações de compromisso social; (3) aprimorar a gestão acadêmica, administrativa e financeira.

Neste sentido, é que se elabora uma série de reflexões novas, reforçando a importância do uso dos resultados já obtidos até então, quanto à avaliação e ao desenvolvimento do trabalho realizado, sobre as bases de um modelo que se mostrou como alavanca propulsora tanto para tomadas de decisões no âmbito das ações institucionalizadas, como para 0 atendimento às especificidades dos diferentes Cursos de Graduação na perspectiva de seu aprimoramento.

\section{Referências}

CONAES. Instrumento de Avaliação de cursos de graduação. Brasília, DF: INEP, 2010, p. 10.

FELDM ANN, M . G. Formação de professores e cotidiano escolar. In: FELDM ANN, M. G. (Org.). Formação de professores e escola na contemporaneidade. São Paulo: Editora SENAC, 2009.

FERNANDES, A. S. Contextos da intervenção educativa local e a experiência dos municípios portugueses em J oão Formosinho. In: FERNANDES, A. S.; MACHADO, J; FERREIRA, F. I. Administração da Educação: lógicas burocráticas e lógicas de mediação. Porto: Edições Asa, 2005. p. 193-221.

MATUS, C. Política: planejamento e governo. Brasília, DF: Editora IPEA, 1993.

PONTIFÍCIA UNIVERSIDADE CATÓLICA DE SÃO PAULO. Estatuto da Pontifícia Universidade Católica de São Paulo. São Paulo: PUC, 2009a.

- Regimento Geral da Pontifícia Universidade Católica de São Paulo. São Paulo: PUC, 2009b.

- Relatório Comissão Própria de Avaliação: (CPA): autoavaliação institucional da PUC-SP: Ciclo 2007/2008. São Paulo: PUC, 2009C.

Recebido em: 21/03/2012

Aceito para publicação em: 12/11/2012 